TRANSACTIONS OF THE

AMERICAN MATHEMATICAL SOCIETY

Volume 358, Number 11, November 2006, Pages 4873-4893

S 0002-9947(06)04050-5

Article electronically published on June 9, 2006

\title{
HARNACK INEQUALITIES AND GAUSSIAN ESTIMATES FOR A CLASS OF HYPOELLIPTIC OPERATORS
}

\author{
ANDREA PASCUCCI AND SERGIO POLIDORO
}

\begin{abstract}
We prove a global Harnack inequality for a class of degenerate evolution operators by repeatedly using an invariant local Harnack inequality. As a consequence we obtain an accurate Gaussian lower bound for the fundamental solution for some meaningful families of degenerate operators.
\end{abstract}

\section{INTRODUCTION}

In this paper we consider a class of linear second order operators in $\mathbb{R}^{N+1}$ of the form

$$
L=\sum_{p=1}^{m} X_{p}^{2}+X_{0}-\partial_{t} .
$$

In (1.1) the $X_{p}$ 's are smooth vector fields on $\mathbb{R}^{N}$, i.e. denoting $z=(x, t)$ as the point in $\mathbb{R}^{N+1}$

$$
X_{p}(x)=\sum_{j=1}^{N} a_{j}^{p}(x) \partial_{x_{j}}, \quad p=0, \ldots, m,
$$

where any $a_{j}^{p}$ is a $C^{\infty}$ function. For our purposes, in the sequel we also consider the $X_{p}$ 's as vector fields in $\mathbb{R}^{N+1}$, and we denote

$$
Y=X_{0}-\partial_{t} \quad \text { and } \quad \lambda \cdot X \equiv \lambda_{1} X_{1}+\cdots+\lambda_{m} X_{m}
$$

for $\lambda=\left(\lambda_{1}, \ldots, \lambda_{m}\right) \in \mathbb{R}^{m}$. We say that a curve $\gamma:[0, T] \rightarrow \mathbb{R}^{N+1}$ is L-admissible if it is absolutely continuous and satisfies

$$
\gamma^{\prime}(s)=\lambda(s) \cdot X(\gamma(s))+Y(\gamma(s)), \quad \text { a.e. in }[0, T],
$$

for suitable piecewise constant real functions $\lambda_{1}, \ldots, \lambda_{m}$. We next state our main assumptions:

[H.1] there exists a homogeneous Lie group $\mathbb{G}=\left(\mathbb{R}^{N+1}, \circ, \delta_{\lambda}\right)$ such that

(i) $X_{1}, \ldots, X_{m}, Y$ are left translation invariant on $\mathbb{G}$;

(ii) $X_{1}, \ldots, X_{m}$ are $\delta_{\lambda}$-homogeneous of degree one, and $Y$ is $\delta_{\lambda}$-homogeneous of degree two;

[H.2] for every $(x, t),(\xi, \tau) \in \mathbb{R}^{N+1}$ with $t>\tau$, there exists an $L$-admissible path $\gamma:[0, T] \rightarrow \mathbb{R}^{N+1}$ such that $\gamma(0)=(x, t), \gamma(T)=(\xi, \tau)$.

Received by the editors August 30, 2004.

2000 Mathematics Subject Classification. Primary 35K57, 35K65, 35K70.

This investigation was supported by the University of Bologna. Funds for selected research topics. 
In the next section we recall the definition and the main properties of homogeneous Lie groups.

Operators of the form (1.1), verifying assumptions [H.1]-[H.2], have been introduced by Kogoj and Lanconelli in [7] and [8]. Under these hypotheses the Hörmander condition holds:

$$
\operatorname{rank} \operatorname{Lie}\left\{X_{1}, \ldots, X_{m}, Y\right\}(z)=N+1, \quad \forall z \in \mathbb{R}^{N+1} ;
$$

hence $L$ in (1.1) is hypoelliptic (i.e. every distributional solution to $L u=0$ is smooth; see, for instance, Proposition 10.1 in [7]) and has a fundamental solution $\Gamma$ which is smooth out of the pole and $\delta_{\lambda}$-homogeneous of degree $2-Q$ :

$$
\Gamma\left(\delta_{\lambda} z\right)=\ell^{2-Q} \Gamma(z), \quad \ell>0
$$

(here $Q$ denotes the homogeneous dimension of $\mathbb{G}$; see Section 21). Hence operator (1.1) belongs to the general class of hypoelliptic operators on homogeneous groups first studied by Folland [4, Rothschild and Stein [18, Nagel, Stein and Wainger 13 .

An invariant (local) Harnack inequality for $L$ is proved in [7, and one-side Liouville theorems are given in $[8$. The main goal of this note is to prove the following non-local Harnack inequality.

Theorem 1.1. Let $z_{0}=\left(x_{0}, t_{0}\right) \in \mathbb{R}^{N+1}$ and $s>0$. There exist two constants $c, C>1$, only dependent on $L$, such that

$$
u\left(\exp (s(\lambda \cdot X+Y))\left(z_{0}\right)\right) \leq C^{1+s|\lambda|^{2}} u\left(z_{0}\right),
$$

for every non-negative solution $u$ to $L u=0$ in $\left.\left.\mathbb{R}^{N} \times\right] t_{0}-c s, t_{0}\right], \lambda \in \mathbb{R}^{m}$.

The connectivity assumption [H.2] and Theorem 1.1 directly yield a global Harnack inequality for positive solutions to $L u=0$ of the form:

$$
u(x, t) \leq H(x, t, \xi, \tau) u(\xi, \tau), \quad \forall(x, t),(\xi, \tau) \mathbb{R}^{N+1}, t<\tau .
$$

When we are able to explicitly find an $L$-admissible path $\gamma$ connecting $(x, t)$ to $(\xi, \tau)$, then we can explicitly express $H(x, t, \xi, \tau)$ and obtain a more useful estimate. Aiming to take into account the homogeneous structure of the Lie group, we construct such a $\gamma$ by separately considering the commutators of different homogeneity of $X_{1}, \ldots, X_{m}, Y$. We remark that these commutators can be conveniently approximated by $L$-admissible paths: for instance, the direction of the commutator $\left[X_{p}, X_{q}\right]$ can be obtained by using the integral curves of $X_{p}, X_{q},-X_{p},-X_{q}$. To be more specific, by using the Campbell-Hausdorff formula, we have

$$
e^{X_{p}+Y} \circ e^{X_{q}+Y} \circ e^{-X_{p}+Y} \circ e^{-X_{q}+Y}=e^{4 Y+\left[X_{p}, X_{q}\right]+R_{2}},
$$

where the error term $R_{2}$ contains commutators $\delta_{\lambda}$-homogeneous of order greater than two. This fact is well known and has been used by many authors in the study of the regularity of "elliptic" and "parabolic" operators of the form

$$
\sum_{p=1}^{m} X_{p}^{2} \quad \text { and } \quad \sum_{p=1}^{m} X_{p}^{2}-\partial_{t}
$$

respectively. However the study of operator (1.1) involves commutators of the form $\left[X_{p}, Y\right]$ that do not occur in the examples (1.7). In this case, we have to use a different combination of vector fields, namely

$$
e^{X_{p}+Y} \circ e^{-X_{p}+Y}=e^{2 Y+\left[X_{p}, Y\right]+R_{3}},
$$


where $R_{3}$ is an error term of order three. The above argument can be adapted to commutators of higher length and leads to an explicit estimate of $H$ in (1.6). This estimate is given in Section 4. Proposition 4.1, in the case of a Lie algebra of step three. We plan to consider Lie groups of a higher step in a future study.

As a consequence of Proposition 4.1 we get the following lower bound for $\Gamma$.

Proposition 1.2. Let $L$ be the operator in (1.1) on a group of step three and $\Gamma$ its fundamental solution. There exists a positive constant $C$ such that

$$
\Gamma(x, t) \geq \frac{C}{t^{\frac{Q-2}{2}}} \exp \left(-C \frac{|x|_{\mathbb{G}}^{6}}{t^{3}}\right), \quad \forall(x, t) \in \mathbb{R}^{N} \times \mathbb{R}^{+} .
$$

Here $|\cdot|_{\mathbb{G}}$ denotes the homogeneous norm in $\mathbb{G}$ (see Section 2 ).

In the above statement $\Gamma(\cdot)$ denotes the fundamental solution of $L$ with pole at the origin. Due to the left o-invariance of $\Gamma$, we have that $\Gamma(z, \zeta)=\Gamma\left(\zeta^{-1} \circ z\right)$ and a lower bound analogous to (1.8) also holds for $\Gamma(\cdot, \zeta)$.

The above estimate looks rather rough, since it is natural to expect $\frac{|x|_{\mathbb{G}}^{2}}{t}$ in the exponent in (1.8). Indeed the following Gaussian upper bound has been proved by Kogoj and Lanconelli in [7]:

$$
\Gamma(x, t) \leq \frac{C}{t^{\frac{Q-2}{2}}} \exp \left(-\frac{|x|_{\mathbb{G}}^{2}}{C t}\right), \quad \forall x \in \mathbb{R}^{N}, t>0,
$$

with $C$ a positive constant. However it is known that the fundamental solution of the (Kolmogorov) operator $\partial_{x_{1}}^{2}+x_{1} \partial_{x_{2}}-\partial_{t}$ is

$$
\Gamma\left(x_{1}, x_{2}, t\right)=\frac{\sqrt{3}}{2 \pi t^{2}} \exp \left(-\frac{x_{1}^{2}}{t}-3 \frac{x_{1} x_{2}}{t^{2}}-3 \frac{x_{2}^{2}}{t^{3}}\right), \quad x_{1}, x_{2} \in \mathbb{R}, t>0 ;
$$

see (1.19) below. In particular

$$
\Gamma\left(0, x_{2}, t\right)=\frac{\sqrt{3}}{2 \pi t^{2}} \exp \left(-3 \frac{x_{2}^{2}}{t^{3}}\right)=\frac{\sqrt{3}}{2 \pi t^{2}} \exp \left(-3 \frac{\left|\left(0, x_{2}\right)\right|_{\mathbb{G}}^{6}}{t^{3}}\right) .
$$

On the other hand, we have

$$
\Gamma\left(x_{1}, 0, t\right)=\frac{\sqrt{3}}{2 \pi t^{2}} \exp \left(-\frac{x_{1}^{2}}{t}\right)=\frac{\sqrt{3}}{2 \pi t^{2}} \exp \left(-\frac{\left|\left(x_{1}, 0\right)\right|_{\mathbb{G}}^{2}}{t}\right),
$$

so that neither (1.8) nor (1.9) are sharp. However we can hope to sharpen (1.8) at least in some component of $x$. The following example shows that further hypotheses on the operator $L$ are needed to obtain such a result. Consider the operator $\widetilde{L}=$ $X^{2}+Y$ in $\mathbb{R}^{3}$, where

$$
X=\partial_{x_{1}}+3 x_{1}^{2} \partial_{x_{2}} \quad \text { and } \quad Y=x_{1} \partial_{x_{2}}-\partial_{t} .
$$

It is straightforward to verify [H.1], [H.2] for $\widetilde{L}$ (the dilations are $\delta_{\lambda}\left(x_{1}, x_{2}, t\right)=$ $\left.\left(\lambda x_{1}, \ell^{3} x_{2}, \ell^{2} t\right)\right)$. The fundamental solution is $\widetilde{\Gamma}\left(x_{1}, x_{2}, t\right)=\Gamma\left(x_{1}, x_{2}-x_{1}^{3}, t\right)$ with $\Gamma$ in (1.10); then

$$
\widetilde{\Gamma}\left(x_{1}, 0, t\right)=\frac{\sqrt{3}}{2 \pi t^{2}} \exp \left(-\frac{\left|x_{1}\right|^{2}}{t}-3 \frac{\left|x_{1}\right|^{4}}{t^{2}}-3 \frac{\left|x_{1}\right|^{6}}{t^{3}}\right), \quad \forall\left(x_{1}, t\right) \in \mathbb{R} \times \mathbb{R}^{+} .
$$

We close the introduction with some examples of operators which verify assumptions [H.1], [H.2] and motivate our study. In these particular cases we will give sharp 
estimates for the case of a Lie algebra of step three (see Propositions $5.1,5.2$ and 5.3 below).

Example 1.3 (Heat operators on Carnot groups). Consider the operator $L$ in (1.1) under assumptions [H.1] and

$$
\operatorname{rank} \operatorname{Lie}\left\{X_{1}, \ldots, X_{m}\right\}(x)=N, \quad \forall x \in \mathbb{R}^{N} .
$$

In this case $\mathbb{G}=\left(\mathbb{R}^{N}, \circ, \delta_{\lambda}\right)$ is a Carnot (or stratified) group (see, for instance, [4] and [20]).

A general result from control theory asserts that, under assumption [H.1], condition (1.13) implies [H.2] (see 6], Theorem 2(a) in Section 4). When $X_{0} \equiv 0$, the couples of conditions [H.1]-[H.2] and [H.1]-(1.13) are equivalent (recall that (1.3) follows from [H.1]-[H.2]). In this case the operator $L$ in (1.1) is

$$
L=\triangle_{\mathbb{G}}-\partial_{t},
$$

where as usual $\triangle_{\mathbb{G}}$ denotes the canonical sub-Laplacian on $\mathbb{G}$ :

$$
\triangle_{\mathbb{G}}=\sum_{p=1}^{m} X_{p}^{2}
$$

We recall the well-known Gaussian upper and lower bounds for heat kernels due to Jerison and Sánchez-Calle [5], Kusuoka and Stroock [10, Varopoulos, Saloff-Coste and Coulhon [20]. These results apply to Lie groups which are not necessarily homogeneous. We also quote the more recent and accurate estimates by SaloffCoste and Stroock [19, Bonfiglioli, Lanconelli and Uguzzoni [3].

More generally condition (1.13) means that the operator (1.1) has the form

$$
L=\triangle_{\mathbb{G}}+X_{0}-\partial_{t},
$$

with $X_{0} \in \operatorname{Lie}\left\{X_{1}, \ldots, X_{m}\right\}$. The results in [5] and [10] apply to the above operator when $X_{0} \in \operatorname{span}\left\{X_{p},\left[X_{q}, X_{r}\right] \mid p, q, r=1, \ldots, m\right\}$ (note that, by the homogeneity assumption [H.1], condition (1.13) implies $\left.X_{0} \in \operatorname{span}\left\{\left[X_{p}, X_{q}\right] \mid p, q=1, \ldots, m\right\}\right)$. Operator (1.15), without other assumptions on $X_{0}$, has been considered by Alexopoulos in [1].

Example 1.4 (Kolmogorov-type operators). Assume $X_{p}=\partial_{p}, p=1, \ldots, m$, and the coefficients of $X_{0}$ are linear functions of $x \in \mathbb{R}^{N}$ :

$$
X_{0}=\langle x, B \nabla\rangle
$$

for a constant $N \times N$ matrix $B$. Then

$$
L=\sum_{p=1}^{m} \partial_{p}^{2}+X_{0}-\partial_{t} .
$$

This kind of operator has been extensively studied (see [11] and [12] for a comprehensive bibliography). It is known that [H.1]-[H.2] for $L$ are equivalent to the following hypothesis:

[H.3] the matrix $B$ takes the form

$$
B=\left(\begin{array}{ccccc}
0 & B_{1} & 0 & \cdots & 0 \\
0 & 0 & B_{2} & \cdots & 0 \\
\vdots & \vdots & \vdots & \ddots & \vdots \\
0 & 0 & 0 & \cdots & B_{n} \\
0 & 0 & 0 & \cdots & 0
\end{array}\right)
$$


for some basis of $\mathbb{R}^{N}$, where $B_{k}$ is a $d_{k} \times d_{k+1}$ matrix of rank $d_{k}, k=$ $1,2, \ldots, n$, with $m=d_{1} \geq d_{2} \geq \cdots \geq d_{n+1} \geq 1$ and $d_{1}+\cdots+d_{n+1}=N$.

The equivalence of [H.3] and the couple of hypotheses [H.1]-(1.3) has been proved in [11. As said before [H.1]-[H.2] yield [H.1]-(1.3); on the other hand in [17] it is proved to be the converse implication for Kolmogorov operators.

Under assumption [H.3], the dilations are

$$
\delta_{\lambda}=\operatorname{diag}\left(\lambda I_{d_{1}}, \ell^{3} I_{d_{2}}, \ldots, \ell^{2 n+1} I_{d_{n+1}}, \ell^{2}\right), \quad \lambda>0,
$$

where $I_{d_{k}}$ is the $d_{k} \times d_{k}$ identity matrix. Moreover the fundamental solution of $L$ in (1.1) is explicitly known:

$$
\Gamma(z)=\frac{1}{\sqrt{(4 \pi)^{N} \operatorname{det} \mathcal{C}(t)}} \exp \left(-\frac{1}{4}\left\langle\mathcal{C}^{-1}(t) x, x\right\rangle\right)
$$

for $t>0$, and $\Gamma(z)=0$ for $t \leq 0$. In (1.19), we denote

$$
E(t)=\exp \left(-t B^{T}\right) \quad \text { and } \quad \mathcal{C}(t)=\int_{0}^{t} E(s) A E^{T}(s) d s,
$$

where $B^{T}$ is the transpose matrix of $B$. We remark that condition [H.3] ensures that $\mathcal{C}(t)>0$ for any $t>0$ (cf. Proposition A.1 in [11]; see also [9]). In this case the group law is

$$
(x, t) \circ(\xi, \tau)=(\xi+E(\tau) x, t+\tau), \quad(x, t),(\xi, \tau) \in \mathbb{R}^{N+1} .
$$

In the sequel we call $\mathbb{K} \equiv\left(\mathbb{R}^{N+1}, \circ\right)$ a Kolmogorov group.

Non-local Harnack inequalities for this kind of operator are proved in [14, moreover Gaussian estimates for the fundamental solution are given in [16], [17] and [15] in the case of non-constant coefficients of the second-order derivatives.

More general examples of operators of the form (1.16) with polynomial (nonlinear) coefficients are

$$
L_{1}=\partial_{x_{1}}^{2}+\partial_{x_{2}}^{2}+x_{1} \partial_{x_{3}}+x_{1} x_{2} \partial_{x_{4}}-\partial_{t}, \quad L_{2}=\partial_{x_{1}}^{2}+x_{1} \partial_{x_{2}}+x_{1}^{2} \partial_{x_{3}}-\partial_{t} .
$$

Note that although both $L_{1}$ and $L_{2}$ satisfy [H.1] and the well-known hypoellipticity Hörmander condition, only $L_{1}$ fulfills [H.2].

Example 1.5 (Operators on linked groups). Let $\mathbb{L}=\mathbb{G} \triangle \mathbb{K}$ be the linked group of a Carnot group $\mathbb{G}$ on $\mathbb{R}^{m} \times \mathbb{R}^{n}$ and a Kolmogorov group $\mathbb{K}$ on $\mathbb{R}^{m} \times \mathbb{R}^{r} \times \mathbb{R}$, as defined by Kogoj and Lanconelli in [7] (Sect. 10). We consider the operator

$$
L=\Delta_{\mathbb{G}}+Y .
$$

For the reader's convenience, we recall the definition of the link of Carnot and Kolmogorov groups. Consider a Carnot group

$$
\mathbb{G}=\left(\mathbb{R}^{m} \times \mathbb{R}^{n}, \circ, \delta_{\lambda}^{\mathbb{G}}\right),
$$

where $(x, y)$ denotes the point in $\mathbb{R}^{m} \times \mathbb{R}^{n}$ and assume that

$$
X_{p}=\partial_{p}+a^{p}(x, y) \nabla_{y}, \quad p=1, \ldots, m .
$$

Hence the dilations and the group law take the following form:

$$
\delta_{\lambda}^{\mathbb{G}}(x, y)=\left(\lambda x, \rho_{\lambda}^{\mathbb{G}} y\right), \quad(x, y) \circ\left(x^{\prime}, y^{\prime}\right)=\left(x+x^{\prime}, Q\left(x, y, x^{\prime}, y^{\prime}\right)\right) .
$$


Moreover the Kolmogorov group is 1

$$
\mathbb{K}=\left(\mathbb{R}^{m} \times \mathbb{R}^{r} \times \mathbb{R}, \circ, \delta_{\lambda}^{\mathbb{K}}\right),
$$

where we denote $(x, w, t)$ as the point in $\mathbb{R}^{m} \times \mathbb{R}^{r} \times \mathbb{R}$. We assume that

$$
Y=X_{0}(x, w)-\partial_{t}=\left\langle(x, w), B \nabla_{(x, w)}\right\rangle-\partial_{t} .
$$

The dilations (1.18) and the group law (1.21) will be denoted by

$$
\begin{gathered}
\delta_{\lambda}^{\mathbb{K}}(x, w, t)=\left(\lambda x, \rho_{\lambda}^{\mathbb{K}} w, \ell^{2} t\right), \\
(x, w, t) \circ\left(x^{\prime}, w^{\prime}, t^{\prime}\right)=\left(x+x^{\prime}, R\left(x, w, t, x^{\prime}, w^{\prime}, t^{\prime}\right), t+t^{\prime}\right) .
\end{gathered}
$$

The link $\mathbb{L}=\mathbb{G} \triangle \mathbb{K}$ is defined as follows:

$$
\mathbb{L}=\left(\mathbb{R}^{m} \times \mathbb{R}^{n} \times \mathbb{R}^{r} \times \mathbb{R}, \circ, \delta_{\lambda}^{\mathbb{L}}\right),
$$

where

and

$$
\delta_{\lambda}^{\mathbb{L}}(x, y, w, t)=\left(\lambda x, \rho_{\lambda}^{\mathbb{G}} y, \rho_{\lambda}^{\mathbb{K}} w, \ell^{2} t\right)
$$

$$
(x, y, w, t) \circ\left(x^{\prime}, y^{\prime}, w^{\prime}, t^{\prime}\right)=\left(x+x^{\prime}, Q\left(x, y, x^{\prime}, y^{\prime}\right), R\left(x, w, t, x^{\prime}, w^{\prime}, t^{\prime}\right), t+t^{\prime}\right) .
$$

It turns out that $\mathbb{L}$ is a homogeneous group, and that the $X_{p}$ 's and $Y$ (considered as vector fields on $\mathbb{R}^{m} \times \mathbb{R}^{n} \times \mathbb{R}^{r} \times \mathbb{R}$ ) satisfy [H.1]-[H.2] (see Propositions 10.4 and 10.5 in [7]). Let us explicitly note that the operations defined in $\mathbb{L}$ extend the ones in $\mathbb{G}$ and $\mathbb{K}$. In particular we have

$$
(x, y, 0,0) \circ\left(x^{\prime}, y^{\prime}, 0,0\right)=\left((x, y) \circ\left(x^{\prime}, y^{\prime}\right), 0,0\right) .
$$

\section{Preliminaries}

In this section we briefly recall the basic properties of homogeneous Lie groups and exponential mappings.

A Lie group $\mathbb{G}=\left(\mathbb{R}^{N+1}, \circ\right)$ is called homogeneous if a family of dilations $\left(\delta_{\lambda}\right)_{\lambda>0}$ exists on $\mathbb{G}$. In our setting, hypotheses [H.1]-[H.2] imply that $\mathbb{R}^{N}$ has a direct sum decomposition

$$
\mathbb{R}^{N}=V_{1} \oplus \cdots \oplus V_{n}
$$

such that, if $x=x^{(1)}+\cdots+x^{(n)}$ with $x^{(k)} \in V_{k}$, then the dilations are

$$
\delta_{\lambda}\left(x^{(1)}+\cdots+x^{(n)}, t\right)=\left(\lambda x^{(1)}+\cdots+\ell^{n} x^{(n)}, \ell^{2} t\right),
$$

for any $(x, t) \in \mathbb{R}^{N+1}$ and $\lambda>0$. We may assume that

$$
\begin{aligned}
x^{(1)} & =\left(x_{1}, \ldots, x_{m_{1}}, 0, \ldots, 0\right) \in V_{1}, \\
x^{(k)} & =\left(0, \ldots, 0, x_{1}^{(k)}, \ldots, x_{m_{k}}^{(k)}, 0, \ldots, 0\right) \in V_{k},
\end{aligned}
$$

for some basis of $\mathbb{R}^{N}$, where

$$
x_{i}^{(k)}=x_{m_{1}+\cdots+m_{k-1}+i}, \quad i=1, \ldots, m_{k} \equiv \operatorname{dim} V_{k} .
$$

The natural number

$$
Q=\sum_{k=1}^{n} k m_{k}+2
$$

\footnotetext{
${ }^{1}$ We use the same notation "o" for the composition law in different groups; the context will avoid ambiguity.
} 
is usually called the homogeneous dimension of $\mathbb{G}$ with respect to $\left(\delta_{\lambda}\right)$. We also introduce the following $\delta_{\lambda}$-homogeneous norms on $\mathbb{R}^{N+1}$ and $\mathbb{R}^{N}$ :

$$
\begin{aligned}
\|(x, t)\|_{\mathbb{G}} & =\left(\sum_{k=1}^{n} \sum_{j=1}^{m_{k}}\left(x_{j}^{(k)}\right)^{\frac{2 n !}{k}}+|t|^{n !}\right)^{\frac{1}{2 n !}}, \\
|x|_{\mathbb{G}} & =\max \left\{\left|x_{i}^{(k)}\right|^{\frac{1}{k}} \mid k=1, \ldots, n, i=1, \ldots, m_{k}\right\} .
\end{aligned}
$$

Since $X_{1}, \ldots, X_{m}$ and $Y$ are smooth vector fields which are $\delta_{\lambda}$-homogeneous, respectively, of degree one and two, it is not difficult to show that they must be of the form

$$
\begin{aligned}
X_{p} & =\sum_{k=1}^{n} a_{k-1}^{p}\left(x^{(1)}, \ldots, x^{(k-1)}\right) \cdot \nabla^{(k)}, \quad p=1, \ldots, m, \\
Y & =\sum_{k=2}^{n} b_{k-2}\left(x^{(1)}, \ldots, x^{(k-2)}\right) \cdot \nabla^{(k)}-\partial_{t}
\end{aligned}
$$

where

$$
\nabla^{(k)}=\left(0, \ldots, 0, \partial_{x_{1}^{(k)}}, \ldots, \partial_{x_{m_{k}}^{(k)}}, 0, \ldots, 0\right)
$$

and $a_{k}^{p}$ and $b_{k}$ are $\delta_{\lambda}$-homogeneous polynomial functions of degree $k$ with values in $V_{k+1}$ and $V_{k+2}$, respectively. Let us explicitly note that hypothesis [H.2] and formula (2.2) imply that $\operatorname{span}\left\{X_{1}(0), \ldots, X_{m}(0)\right\}=V_{1}$. Then we may assume $m=m_{1}$ and $X_{p}(0)=\mathbf{e}_{p}$ for $p=1, \ldots, m$, where $\left\{\mathbf{e}_{i}\right\}_{1 \leq i \leq N}$ denotes the canonical basis of $\mathbb{R}^{N}$. We also remark that, by [H.2] and (2.2), $\operatorname{span}\left\{X_{0}(0),\left[X_{p}, X_{q}\right](0)\right\}=$ $V_{2}$; then, in the particular case of a Kolmogorov group, $V_{2}=\{0\}$ necessarily.

We denote by $\mathfrak{g}$ the Lie algebra of $\mathbb{G}$. For any $X \in \mathfrak{g}, z \in \mathbb{R}^{N+1}$ and $s \in \mathbb{R}$, we let $\exp (s X)(z)=\gamma(s)$, where $\gamma$ is the (unique and globally defined) solution to the Cauchy problem

$$
\gamma^{\prime}=X(\gamma), \quad \gamma(0)=z
$$

We also use the following notation $e^{X}=\exp (X)(0)$ and recall that

$$
\exp (X)(z)=z \circ e^{X}, \quad \forall X \in \mathfrak{g}, z \in \mathbb{R}^{N+1} .
$$

In the sequel we use the well-known Campbell-Hausdorff formula which we recall here for greater convenience:

$$
e^{X} \circ e^{Z}=e^{X+Z+\frac{1}{2}[X, Z]+\frac{1}{12}[X-Z,[X, Z]]+R_{3}},
$$

where $R_{3}$ are commutators of length greater than three.

\section{Proof of Theorem 1.1}

We prove Theorem 1.1 by constructing a Harnack chain by means of the Harnack inequality proved in [7. We recall that a set $\left\{z_{0}, \ldots, z_{k}\right\} \subseteq O$, where $O$ is an open subset of $\mathbb{R}^{N+1}$, is said a Harnack chain of length $k$ if a positive constant $C$ exists such that

$$
u\left(z_{j}\right) \leq C u\left(z_{j-1}\right), \quad \text { for } j=1, \ldots, k,
$$

for every non-negative solution $u$ to $L u=0$ in $O$.

We choose $\left\{z_{0}, \ldots, z_{k}\right\}$ along a suitable integral curve of the vector field $\lambda \cdot X+Y$. The technique goes back to Aronson and Serrin [2 for the study of uniformly 
parabolic equations. Then it has been extended in 20] to the framework of heat kernels on Lie groups and in [17] to Kolmogorov groups.

We first recall the invariant Harnack inequality proved in 7, Theorem 7.1. Given $r>0, \varepsilon \in] 0,1\left[\right.$ and $z_{0} \in \mathbb{R}^{N+1}$, we put

$$
\mathcal{C}_{r}\left(z_{0}\right)=z_{0} \circ \delta_{r}\left(\mathcal{C}_{1}\right), \quad \mathcal{S}_{r}^{(\varepsilon)}\left(z_{0}\right)=z_{0} \circ \delta_{r}\left(\mathcal{S}_{1}^{(\varepsilon)}\right),
$$

where

$\mathcal{C}_{1}=\left\{z=(x, t) \in \mathbb{R}^{N+1} \mid\|z\|_{\mathbb{G}} \leq 1, t \leq 0\right\}, \quad \mathcal{S}_{1}^{(\varepsilon)}=\left\{z=(x,-\varepsilon) \mid z \in \mathcal{C}_{1}\right\}$.

We remark that the following result is stated in [7] only for $\varepsilon \in\left[\frac{1}{4}, \frac{3}{4}\right]$, however the compactness argument used in the proof applies to every $\varepsilon \in] 0,1[$.

Theorem 3.1. Let $O$ be an open set in $\mathbb{R}^{N+1}$ containing $\mathcal{C}_{r}\left(z_{0}\right)$ for some $z_{0} \in \mathbb{R}^{N+1}$ and $r>0$. Given $\varepsilon \in] 0,1[$, there exist two positive constants $\theta=\theta(L, \varepsilon)$ and $C=C(L, \varepsilon)$ such that

$$
\sup _{\mathcal{S}_{\theta r}^{(\varepsilon)}\left(z_{0}\right)} u \leq C u\left(z_{0}\right)
$$

for every non-negative solution u of $L$ in $O$.

We next prove a different version of the above Harnack inequality.

Proposition 3.2. Let $O$ be an open set in $\mathbb{R}^{N+1}$ containing $\mathcal{C}_{r}\left(z_{0}\right)$ for some $z_{0} \in$ $\mathbb{R}^{N+1}$ and $r>0$. For every $R>0$ there exist two positive constants $C, \bar{c}$ only dependent on $L$ and $R$ such that

$$
u\left(z_{0} \circ z\right) \leq C u\left(z_{0}\right)
$$

for every non-negative solution $u$ of $L$ in $O$ and for every $z$ in the paraboloid

$$
\mathcal{P}_{R, T}=\left\{\left.(x,-t) \in \mathbb{R}^{N+1}|| x\right|_{\mathbb{G}} ^{2} \leq R t, 0<t \leq T\right\},
$$

where $0<T \leq \bar{c} r^{2}$.

Proof. For a fixed $t>0$, we put

$$
A_{t}=\left\{\left.(x,-t)|| x\right|_{\mathbb{G}} ^{2}<R t\right\} .
$$

For every $z \in A_{t}$ we have

$$
\|z\|_{\mathbb{G}}^{2} \leq\left(N|x|_{\mathbb{G}}^{2 \sigma}+t^{\sigma}\right)^{\frac{1}{\sigma}}<c_{R} t
$$

with $c_{R}=\left(1+N R^{\sigma}\right)^{\frac{1}{\sigma}}$, therefore

$$
A_{t} \subseteq\left\{z=(x,-t) \mid\|z\|^{2}<c_{R} t\right\}=\mathcal{S}_{\sqrt{c_{R} t}}^{\left(c_{R}^{-1}\right)} .
$$

Since $c_{R}>1$, we can apply Theorem 3.1 with $\varepsilon=c_{R}^{-1}$. Then there exist $\theta, C>0$, only dependent on $L$ and $R$, such that

$$
\sup _{\mathcal{S}_{\rho}^{(\varepsilon)}\left(z_{0}\right)} u \leq C u\left(z_{0}\right)
$$

for every non-negative solution $u$ of $L$ in $O$ and for any $\rho \in] 0, \theta r]$. If we put $T=c_{R}^{-1} \theta^{2} r^{2}$ we have $A_{t} \subseteq \mathcal{S}_{\rho}^{(\varepsilon)}$ with $\rho=\sqrt{c_{R} t} \leq \theta r$ when $t \leq T$. The claim follows from (3.5).

The previous proposition states a Harnack inequality for a paraboloid of arbitrarily large width $R$ : we next make a suitable choice of $R$ in order to use (3.3) in the proof of Theorem 1.1 
Proposition 3.3. There exists $R>1$ only dependent on $L$ such that

$$
\exp (s(\lambda \cdot X+Y))(0) \in \mathcal{P}_{R,|\lambda|^{-2}}
$$

for any $\lambda \in \mathbb{R}^{m}, \lambda \neq 0$, and $\left.\left.s \in\right] 0,|\lambda|^{-2}\right]$. The statement also holds for $\lambda=0$ : in this case $\exp (s Y)(0) \in \mathcal{P}_{R, T}$ for any $s, T>0$ with $s \leq T$.

Proof. If $\lambda \neq 0$, we set

$$
\exp (s(\lambda \cdot X+Y))(0)=(\gamma(s),-s),
$$

and recall the expressions (2.2) of the vector fields $X_{p}$ and $Y$. We aim to show that under the condition

$$
0<s|\lambda|^{2} \leq 1,
$$

then $(\gamma(s),-s) \in \mathcal{P}_{R,|\lambda|^{-2}}$ for some suitable $R>1$ only dependent on $L$, or in other terms

$$
\left|\gamma_{i}^{(k)}(s)\right|^{\frac{2}{k}}<R s, \quad i=1, \ldots, m_{k}, \quad k=1, \ldots, n .
$$

We have $\gamma^{(1)}(s)=s \lambda$, so that

$$
\left|\gamma^{(1)}(s)\right|^{2}=s^{2}|\lambda|^{2}<R s
$$

by (3.7) provided that $R>1$. This proves the case $k=1$ in (3.8). Next, we have

$$
\dot{\gamma}^{(2)}(s)=\sum_{p=1}^{m} \lambda_{p} a_{1}^{p}\left(\gamma^{(1)}(s)\right)+b_{0} .
$$

Then, for some constant vector $\mathbf{v} \in V_{2}$, we have

$$
\gamma^{(2)}(s)=s\left(s|\lambda|^{2} \mathbf{v}+b_{0}\right)
$$

By (3.7) we may choose $R$, only dependent on $L$, large enough so that

$$
\left|\gamma_{i}^{(2)}(s)\right| \leq R s, \quad i=1, \ldots, m_{2},
$$

which proves the case $k=2$ in (3.8). For $3 \leq k \leq n$, we have

$$
\dot{\gamma}^{(k)}(s)=\sum_{p=1}^{m} \lambda_{p} a_{k-1}^{p}\left(\gamma^{(1)}(s), \ldots, \gamma^{(k-1)}(s)\right)+b_{k-2}\left(\gamma^{(1)}(s), \ldots, \gamma^{(k-2)}(s)\right),
$$

and, since $a_{k}^{p}$ and $b_{k}$ are $\delta$-homogeneous functions of degree $k$, a straightforward inductive argument yields

$$
\gamma^{(k)}(s)=s^{\frac{k}{2}}\left(\mathbf{v}_{0}+\left(s|\lambda|^{2}\right)^{\frac{1}{2}} \mathbf{v}_{1}+\cdots+\left(s|\lambda|^{2}\right)^{\frac{k}{2}} \mathbf{v}_{k}\right)
$$

for some constant vectors $\mathbf{v}_{j}$ belonging to $V_{k}$ and only dependent on $L$. Therefore, by (3.7) we may enlarge $R=R(L)$ if necessary, so that (3.8) holds. The same argument applies to the case $\lambda=0$.

We are now in a position to prove Theorem 1.1. Note that, in the statement, the domain of the solution $u$ is the strip $\left.\left.\mathbb{R}^{N} \times\right] t_{0}-c s, t_{0}\right]$; here we prove inequality (1.5) under a bit less restrictive assumptions. Theorem 1.1 will be a direct consequence of the following Proposition 3.4. The proof is based on the construction of a Harnack chain along the path

$$
\gamma(\tau)=\exp (\tau s(\lambda \cdot X+Y))\left(z_{0}\right), \quad \tau \in[0,1],
$$


where $s>0, \lambda \in \mathbb{R}^{m}$ and $z_{0} \in \mathbb{R}^{N+1}$ are given. In view of Proposition 3.3, if $k$ is the natural number such that

$$
\frac{k-1}{|\lambda|^{2}}<s \leq \frac{k}{|\lambda|^{2}}
$$

we consider the set $\left\{z_{0}, \ldots, z_{k}\right\}$ defined as

$$
z_{j}=\exp \left(\frac{j}{|\lambda|^{2}}(\lambda \cdot X+Y)\right)\left(z_{0}\right), \quad j=1, \ldots, k-1,
$$

and $z_{k}=\exp (s(\lambda \cdot X+Y))\left(z_{0}\right)$, and we will show that it is a Harnack chain.

Proposition 3.4. Let $O$ be a domain of $\mathbb{R}^{N+1}, z_{0} \in O, \lambda \in \mathbb{R}^{m}$ and $s>0$. With $\bar{c}, C$ being the constants in Proposition 3.2 , we set $r=\min \left\{\frac{1}{|\lambda| \sqrt{\bar{c}}}, \sqrt{\frac{s}{\bar{c}}}\right\}$. Suppose that $\mathcal{C}_{r}\left(z_{j}\right) \subseteq O$ for $j=0, \ldots, k-1$, where the set $\left\{z_{0}, \ldots, z_{k}\right\}$ is defined in (3.10). Then

$$
u\left(\exp (s(\lambda \cdot X+Y))\left(z_{0}\right)\right) \leq C^{1+s|\lambda|^{2}} u\left(z_{0}\right),
$$

for every non-negative solution $u$ to $L u=0$ in $O$.

Proof. We first suppose $s \leq \frac{1}{|\lambda|^{2}}$ : in this case $s=\bar{c} r^{2}$ and $k=1$, then we apply Proposition 3.2 once in view of (3.6).

Now suppose $s>\frac{1}{|\lambda|^{2}}$. We consider the set $\left\{z_{0}, \ldots, z_{k}\right\}$ defined in (3.10), and we show that it is a Harnack chain. To this aim it suffices to note that

$$
z_{j}=z_{j-1} \circ \exp \left(\frac{1}{|\lambda|^{2}}(\lambda \cdot X+Y)\right)(0), \quad j=1, \ldots, k-1,
$$

and to recall (3.6). Then we apply Proposition $3.2 k$ times since in this case $|\lambda|^{2}=\bar{c} r^{2}$ and $\mathcal{C}_{r}\left(z_{j}\right) \subseteq O$, by our hypothesis. Note that $k<1+s|\lambda|^{2}$ by (3.9) and the thesis easily follows.

Proof of Theorem 1.1. We consider the set $\left\{z_{0}, \ldots, z_{k}\right\}$ defined in (3.10), and we note that $\left.\left.\mathcal{C}_{r}\left(z_{j}\right) \subseteq \mathbb{R}^{N} \times\right] t_{0}-c s, t_{0}\right]$, for any $j=0, \ldots, k-1$, provided that we choose $c \geq 1+\frac{1}{\bar{c}}$. Then the claim follows from Proposition 3.4.

\section{Global Harnack inequalities}

In this section, by repeatedly applying Theorem [1.1 we prove a Harnack inequality for non-negative solutions to $L u=0$ in a strip $\mathbb{R}^{N} \times I$ in the case of a Lie algebra of step three, so that $\mathbb{R}^{N}=V_{1} \oplus V_{2} \oplus V_{3}$. We remark that $\left[X_{r},\left[X_{p}, X_{q}\right]\right]$ and $\left[X_{p}, Y\right]$ are both commutators of order three, but they play a different role in our estimates; hence it is convenient to split $V_{3}$ as $W^{\prime} \oplus W^{\prime \prime}$, where $W^{\prime}=\operatorname{span}\left\{\left[X_{r},\left[X_{p}, X_{q}\right]\right] \mid r, p, q=1, \ldots, m_{1}\right\}, W^{\prime \prime}=V_{3} \cap\left(W^{\prime}\right)^{\perp}$ and, accordingly, $x^{(3)}=w^{\prime}+w^{\prime \prime}$, with $w^{\prime} \in W^{\prime}$ and $w^{\prime \prime} \in W^{\prime \prime}$ (actually, the space $V_{3}$ can be split in other ways, however it seems that this choice yields better estimates; see Remark 4.7 below).

We next state a Harnack inequality for a general operator acting on a Lie algebra of step three. Then some more accurate estimates will be given in Propositions 4.2. 4.3 and 4.4 under geometrical conditions on the Lie group.

Proposition 4.1. Let $L$ be the operator in (1.1) on a group of step three and let $z_{0}=\left(x_{0}, t_{0}\right) \in \mathbb{R}^{N+1}, T>0$. There exist two constants $c>0$ and $C>1$, 
only dependent on $L$, such that, if $u$ is a non-negative solution to $L u=0$ in $\left.\left.\mathbb{R}^{N} \times\right] t_{0}-c T, t_{0}+T\right]$, then

$$
u\left(z_{0}\right) \leq \exp \left(C\left(1+\frac{|x|_{\mathbb{G}}^{6}}{s^{3}}\right)\right) u\left(z_{0} \circ z\right)
$$

for every $\left.\left.z=(x, s) \in \mathbb{R}^{N} \times\right] 0, T\right]$.

The examples (1.11) and (1.12) in the Introduction show that (4.1) cannot be improved in general. We next state some sharper estimates for the operators in Examples 1.3, 1.4 and 1.5.

Proposition 4.2. Let $L$ be a parabolic operator on a Carnot group of step three (cf. Example 1.3) and let $z_{0}=\left(x_{0}, t_{0}\right) \in \mathbb{R}^{N+1}, T>0$. There exist two constants $c>0$ and $C>1$, only dependent on $L$, such that, if $u$ is a non-negative solution to $L u=0$ in $\left.\left.\mathbb{R}^{N} \times\right] t_{0}-c T, t_{0}+T\right]$, then

$$
u\left(z_{0}\right) \leq \exp \left(C\left(1+\frac{|x|_{\mathbb{G}}^{2}}{s}\right)\right) u\left(z_{0} \circ z\right)
$$

for every $\left.\left.z=(x, s) \in \mathbb{R}^{N} \times\right] 0, T\right]$.

Proposition 4.3. Let $L$ be a Kolmogorov-type operator on a group $\mathbb{K}$ of step three (cf. Example 1.4) and let $z_{0}=\left(x_{0}, t_{0}\right) \in \mathbb{R}^{N+1}, T>0$. There exist two constants $c>0$ and $C>1$, only dependent on $L$, such that, if $u$ is a non-negative solution to $L u=0$ in $\left.\left.\mathbb{R}^{N} \times\right] t_{0}-c T, t_{0}+T\right]$, then

$$
u\left(z_{0}\right) \leq \exp \left(C\left(1+\frac{\left|x^{(1)}\right|_{\mathbb{K}}^{2}}{s}+\frac{\left|x^{(3)}\right|_{\mathbb{K}}^{6}}{s^{3}}\right)\right) u\left(z_{0} \circ z\right),
$$

for every $\left.\left.z=(x, s) \in \mathbb{R}^{N} \times\right] 0, T\right]$.

Proposition 4.4. Let $L$ be the operator in (1.22) on a linked group $\mathbb{L}=\mathbb{G} \triangle \mathbb{K}$ of step three. Let $z_{0}=\left(\xi_{0}, \eta_{0}, \omega_{0}, t_{0}\right) \in \mathbb{R}^{N+1} \equiv \mathbb{R}^{m} \times \mathbb{R}^{n} \times \mathbb{R}^{r} \times \mathbb{R}$ and $T>0$. There exist two constants $c>0$ and $C>1$, only dependent on $L$, such that, if $u$ is a non-negative solution to $L u=0$ in $\left.\left.\mathbb{R}^{N} \times\right] t_{0}-c T, t_{0}+T\right]$, then

$$
u\left(z_{0}\right) \leq \exp \left(C\left(1+\frac{|(\xi, \eta, 0)|_{\mathbb{L}}^{2}}{s}+\frac{|(0,0, \omega)|_{\mathbb{L}}^{6}}{s^{3}}\right)\right) u\left(z_{0} \circ z\right),
$$

for every $\left.\left.z=(\xi, \eta, \omega, s) \in \mathbb{R}^{N} \times\right] 0, T\right]$.

Before giving the proof, we introduce some notation and prove some preliminary lemmas. Since the Lie algebra has step three, the vector fields in (2.2) take the form

$$
\begin{aligned}
X_{p} & =\partial_{p}+a_{1}^{p}\left(x^{(1)}\right) \nabla^{(2)}+a_{2}^{p}\left(x^{(1)}, x^{(2)}\right) \nabla^{(3)}, \quad p=1, \ldots, m_{1}, \\
Y & =b_{0} \nabla^{(2)}+b_{1}\left(x^{(1)}\right) \nabla^{(3)}-\partial_{t},
\end{aligned}
$$

with $a_{1}^{p}: V_{1} \longrightarrow V_{2}$ and $b_{1}: V_{1} \longrightarrow V_{3}$ linear functions, $b_{0} \in V_{2}$ and

$$
a_{2}^{p}\left(x^{(1)}, x^{(2)}\right)=A_{2}^{p}\left(x^{(1)}\right)+A_{1}^{p}\left(x^{(2)}\right),
$$

where $A_{2}^{p}: V_{1} \longrightarrow V_{3}$ is a bilinear function and $A_{1}^{p}: V_{2} \longrightarrow V_{3}$ is a linear function, for $p=1, \ldots, m_{1}$. 
Lemma 4.5. Let $z=\left(x-t b_{0}, t\right) \in \mathbb{R}^{N} \times \mathbb{R}^{+}$with $x \in W^{\prime \prime}$ and $b_{0} \in V_{2}$ as in (4.5) 2 There exist two constants $c>0, C>1$, only dependent on $L$, such that

$$
u(0) \leq \exp \left(C\left(1+\frac{|x|^{2}}{t^{3}}\right)\right) u(z)
$$

for every non-negative solution $u$ to $L u=0$ in $\left.\left.\mathbb{R}^{N} \times\right]-c t, t\right]$.

Proof. In order to apply Theorem 1.1 we aim to connect $z$ to 0 by using suitable integral curves of the vector fields $\lambda X_{p}+Y$. This is possible since the CampbellHausdorff formula (2.3) (with $R_{3} \equiv 0$, since we assume that the Lie algebra has step three) yields

$$
e^{X_{p}+Y} \circ e^{-X_{p}+Y}=e^{2 Y+\left[X_{p}, Y\right]} .
$$

To prove our claim, we select a basis

$$
\left\{\left[X_{p_{1}}, Y\right], \ldots,\left[X_{p_{m_{3}^{\prime \prime}}}, Y\right]\right\}
$$

of $W^{\prime \prime}$ and, without loss of generality, we assume that

$$
\left[X_{s_{k}}, Y\right]=\partial_{m_{1}+m_{2}+m_{3}^{\prime}+k} \quad \text { for } k=1, \ldots, m_{3}^{\prime \prime} .
$$

We put $\tau=\frac{t}{2 m_{3}^{\prime \prime}}, z_{0}=z$,

$$
z_{k}=z_{k-1} \circ e^{\tau\left(\lambda_{k} X_{s_{k}}+Y\right)} \circ e^{\tau\left(-\lambda_{k} X_{s_{k}}+Y\right)}, \quad k=1, \ldots, m_{3}^{\prime \prime},
$$

and we choose $\lambda_{k}$ such that $z_{m_{3}^{\prime \prime}}=0$. By (4.8), we have

$$
z_{k}=z_{k-1} \circ e^{2 \tau Y+\tau^{2} \lambda_{k}\left[X_{s_{k}}, Y\right]},
$$

then, by again using the Campbell-Hausdorff formula and (4.9), we get

$$
z_{m_{3}^{\prime \prime}}=z \circ \exp \left(t Y+\sum_{1 \leq k \leq m_{3}^{\prime \prime}} \tau^{2} \lambda_{k}\left[X_{s_{k}}, Y\right]\right)=\left(x+\sum_{1 \leq k \leq m_{3}^{\prime \prime}} \tau^{2} \lambda_{k} \mathbf{e}_{m_{3}^{\prime}+k}^{(3)}, 0\right) .
$$

The claim follows by setting $\lambda_{k}=-\tau^{-2} x_{m_{3}^{\prime}+k}^{(3)}$ in (4.10) and applying Theorem 1.1 repeatedly.

Lemma 4.6. Let $z=\left(x-2 t b_{0}, 2 t\right) \in \mathbb{R}^{N} \times \mathbb{R}^{+}$, with $x \in V_{3}$. There exist two constants $c>0, C>1$, only dependent on $L$, such that

$$
u(\bar{z}) \leq \exp \left(C\left(1+\sum_{1 \leq k \leq m_{3}^{\prime \prime}} \frac{\left|x_{m_{1}+m_{2}+k}\right|^{\frac{2}{3}}}{t}\right)\right) u(z),
$$

for every non-negative solution $u$ to $L u=0$ in $\left.\left.\mathbb{R}^{N} \times\right]-c t, 2 t\right]$. In (4.11)

$$
\bar{z}=\left(\bar{x}-t b_{0}, t\right) \text {, }
$$

where $\bar{x} \in V_{3}$ is such that

$$
\bar{x}_{j}^{(3)}=0, \quad j=1, \ldots, m_{3}^{\prime}, \quad \bar{x}_{j}^{(3)}=x_{j}^{(3)}, \quad j=m_{3}^{\prime}+1, \ldots, m_{3} .
$$

\footnotetext{
${ }^{2}$ The term $-t b_{0}$ in $z$ appears in order to take into account the constant drift of $Y$.
} 
Proof. We connect $z$ to $\bar{z}$ in (4.12) by using a path moving in the direction of $\left[X_{r},\left[X_{p}, X_{q}\right]\right]$ : in order to apply Theorem 1.1 we use suitable integral curves of the vector fields $X_{r}+Y, X_{p}+Y$ and $X_{q}+Y$. Indeed, by the Campbell-Hausdorff formula, we have

$$
\begin{aligned}
e^{4\left(X_{r}+Y\right)} \circ & \left(e^{X_{p}+Y} \circ e^{X_{q}+Y} \circ e^{-X_{p}+Y} \circ e^{-X_{q}+Y}\right) \\
\circ & e^{4\left(-X_{r}+Y\right)} \circ\left(e^{-X_{p}+Y} \circ e^{-X_{q}+Y} \circ e^{X_{p}+Y} \circ e^{X_{q}+Y}\right) \\
& =e^{16 Y+4\left[X_{r},\left[X_{p}, X_{q}\right]\right]+4\left[4 X_{r}+X_{p}+X_{q}, Y\right]} .
\end{aligned}
$$

Arguing as in the proof of Lemma 4.5, we select a basis

$$
\left\{\left[X_{r_{1}},\left[X_{p_{1}}, X_{q_{1}}\right]\right], \ldots,\left[X_{r_{m_{3}^{\prime}}},\left[X_{p_{m_{3}^{\prime}}}, X_{q_{m_{3}^{\prime}}}\right]\right\}\right.
$$

of $W^{\prime}$ and, without loss of generality, assume that

$$
\left[X_{r_{k}},\left[X_{p_{k}}, X_{q_{k}}\right]\right]=\partial_{m_{1}+m_{2}+k} \quad \text { for } k=1, \ldots, m_{3}^{\prime} .
$$

Then we put $\tau=\frac{t}{32 m_{3}^{\prime}}, \bar{z}_{0}=z$ and

$$
\begin{aligned}
\bar{z}_{k}=\bar{z}_{k-1} & \circ e^{4 \tau\left(\lambda_{k} X_{r_{k}}+Y\right)} \\
& \circ\left(e^{\tau\left(\lambda_{k} X_{p_{k}}+Y\right)} \circ e^{\tau\left(\lambda_{k} X_{q_{k}}+Y\right)} \circ e^{\tau\left(-\lambda_{k} X_{p_{k}}+Y\right)} \circ e^{\tau\left(-\lambda_{k} X_{q_{k}}+Y\right)}\right) \\
& \circ e^{4 \tau\left(-\lambda_{k} X_{r_{k}}+Y\right)} \\
& \circ\left(e^{\tau\left(-\lambda_{k} X_{p_{k}}+Y\right)} \circ e^{\tau\left(-\lambda_{k} X_{q_{k}}+Y\right)} \circ e^{\tau\left(\lambda_{k} X_{p_{k}}+Y\right)} \circ e^{\tau\left(\lambda_{k} X_{q_{k}}+Y\right)}\right),
\end{aligned}
$$

for $k=1, \ldots, m_{3}^{\prime}$, where $\lambda_{1}, \ldots, \lambda_{m_{3}^{\prime}}$ will be chosen later. By (4.13), we have

$$
\bar{z}_{k}=\bar{z}_{k-1} \circ e^{16 \tau Y+4 \tau^{3} \lambda_{k}^{3}\left[X_{r_{k}},\left[X_{p_{k}}, X_{q_{k}}\right]\right]+4 \tau^{2} \lambda_{k}\left[X_{p_{k}}+X_{q_{k}}+4 X_{r_{k}}, Y\right]} ;
$$

moreover, by the Campbell-Hausdorff formula and (4.14), we get

$$
\bar{z}_{m_{3}^{\prime}}=\exp \left(\frac{t}{2} Y+4 \tau^{2} \sum_{1 \leq k \leq m_{3}^{\prime}}\left(\tau \lambda_{k}^{3} \mathbf{e}_{k}^{(3)}+\lambda_{k}\left[X_{p_{k}}+X_{q_{k}}+4 X_{r_{k}}, Y\right]\right)\right)(z)
$$

Note that the approximation (4.15) of the commutator $\left[X_{r_{k}},\left[X_{p_{k}}, X_{q_{k}}\right]\right]$ introduces in (4.16) the "error term" $\left[X_{p_{k}}+X_{q_{k}}+4 X_{r_{k}}, Y\right]$. Therefore we rely on (4.8) to remove it: we set $z_{0}=z_{m_{3}^{\prime}}$ and

$$
\begin{aligned}
z_{k}=z_{k-1} & \circ\left(e^{4 \tau\left(-\lambda_{k} X_{r_{k}}+Y\right)} \circ e^{4 \tau\left(\lambda_{k} X_{r_{k}}+Y\right)}\right) \\
& \circ\left(e^{2 \tau\left(-\lambda_{k} X_{p_{k}}+Y\right)} \circ e^{2 \tau\left(\lambda_{k} X_{p_{k}}+Y\right)}\right) \circ\left(e^{2 \tau\left(-\lambda_{k} X_{q_{k}}+Y\right)} \circ e^{2 \tau\left(\lambda_{k} X_{q_{k}}+Y\right)}\right)
\end{aligned}
$$

for $k=1, \ldots, m_{3}^{\prime}$. By (4.8) we obtain

$$
z_{m_{3}^{\prime}}=z \circ \exp \left(t Y+4 \tau^{3} \sum_{1 \leq k \leq m_{3}^{\prime}} \lambda_{k}^{3} \mathbf{e}_{k}^{(3)}\right)=\left(x-t b_{0}+4 \tau^{3} \sum_{1 \leq k \leq m_{3}^{\prime}} \lambda_{k}^{3} \mathbf{e}_{k}^{(3)}, t\right)
$$

Next we choose $\lambda_{k}$ such that

$$
x_{k}^{(3)}+4 \lambda_{k}^{3} \tau^{3}=0, \quad k=1, \ldots, m_{3}^{\prime},
$$

and the claim follows from Theorem 1.1 
Remark 4.7. Consider the vector space

$$
W=\operatorname{span}\left\{\left[X_{r},\left[X_{p}, X_{q}\right]\right] \mid r, p, q=1, \ldots, m_{1}\right\} \cap \operatorname{span}\left\{\left[X_{p}, Y\right] \mid p=1, \ldots, m_{1}\right\}
$$

and suppose that it is non-trivial. Then we can use both (4.8) and (4.13) to obtain an estimate of the form

$$
u(0) \leq C \min \left\{\exp \left(1+\frac{|x|^{2}}{t^{3}}\right), \exp \left(1+\frac{|x|^{\frac{2}{3}}}{t}\right)\right\} u(z),
$$

for $z=\left(x-t b_{0}, t\right) \in \mathbb{R}^{N} \times \mathbb{R}^{+}$with $x \in W$. However this estimate does not improve (4.11).

Lemma 4.8. Let $z=(x, 3 t) \in V_{1}^{\perp} \times \mathbb{R}^{+}$. There exist two constants $c>0, C>1$, only dependent on $L$, such that

$$
u(\bar{z}) \leq \exp \left(C\left(1+\frac{\left|x^{(2)}\right|}{t}\right)\right) u(z),
$$

for every non-negative solution $u$ to $L u=0$ in $\left.\left.\mathbb{R}^{N} \times\right]-c t, 3 t\right]$, where

$$
\bar{z}=\left(\bar{x}-2 t b_{0}, 2 t\right),
$$

with $\bar{x} \in V_{3}, \bar{x}^{(3)}=x^{(3)}$.

Proof. We connect $z$ to $\bar{z}$ in (4.19) by using a combination of paths of the following form:

$$
e^{X_{p}+Y} \circ e^{X_{q}+Y} \circ e^{-X_{p}+Y} \circ e^{-X_{q}+Y}=e^{4 Y+\left[X_{p}, X_{q}\right]+2\left[X_{p}+X_{q}, Y\right]+\frac{1}{2}\left[X_{p}+X_{q},\left[X_{p}, X_{q}\right]\right]} .
$$

Then we select a basis

$$
\left\{\left[X_{p_{1}}, X_{q_{1}}\right], \ldots,\left[X_{p_{m_{2}}}, X_{q_{m_{2}}}\right]\right\}
$$

of $\operatorname{span}\left\{\left[X_{p}, X_{q}\right] \mid p, q=1, \ldots, m_{1}\right\}$, and we assume, since it is not restrictive, that

$$
\left[X_{p_{k}}, X_{q_{k}}\right]=\partial_{m_{1}+k}+a_{1}^{p_{k} q_{k}} \nabla^{(3)}, \quad k=1, \ldots, m_{2},
$$

for some suitable linear functions $a_{1}^{p_{k} q_{k}}$. Next we put $\tau=\frac{t}{40 m_{2}}, z_{0}=z$ and

$$
\begin{aligned}
& z_{k}=z_{k-1} \circ e^{\tau\left(\lambda_{k} X_{p_{k}}+Y\right)} \\
& \circ e^{\tau\left(\lambda_{k} X_{q_{k}}+Y\right)} \circ e^{\tau\left(-\lambda_{k} X_{p_{k}}+Y\right)} \circ e^{\tau\left(-\lambda_{k} X_{q_{k}}+Y\right)}, \quad k=1, \ldots, m_{2},
\end{aligned}
$$

for some $\lambda_{1}, \ldots, \lambda_{m_{2}}$ to be suitably chosen. By (4.20), we have

$$
\begin{aligned}
z_{k}= & \exp \left(4 \tau Y+\tau^{2} \lambda_{k}^{2}\left[X_{p_{k}}, X_{q_{k}}\right]\right. \\
& \left.+2 \tau^{2} \lambda_{k}\left[X_{p_{k}}+X_{q_{k}}, Y\right]+\frac{1}{2} \tau^{3} \lambda_{k}^{3}\left[X_{p_{k}}+X_{q_{k}},\left[X_{p_{k}}, X_{q_{k}}\right]\right]\right)\left(z_{k-1}\right) ;
\end{aligned}
$$

then if we let $\overline{\bar{z}}_{0}=z_{m_{2}}$, we get

$$
\begin{aligned}
\overline{\bar{z}}_{0}= & \exp \left(\frac{t}{10} Y+\sum_{1 \leq k \leq m_{2}} \tau^{2} \lambda_{k}^{2}\left[X_{p_{k}}, X_{q_{k}}\right]\right. \\
& \left.+2 \sum_{1 \leq k \leq m_{2}} \tau^{2} \lambda_{k}\left[X_{p_{k}}+X_{q_{k}}, Y\right]+\frac{1}{2} \sum_{1 \leq k \leq m_{2}} \tau^{3} \lambda_{k}^{3}\left[X_{p_{k}}+X_{q_{k}},\left[X_{p_{k}}, X_{q_{k}}\right]\right]\right)(z)
\end{aligned}
$$


Before choosing the $\lambda_{k}$ 's, we remove the error terms $\left[X_{p_{k}}+X_{q_{k}}, Y\right]$ and $\left[X_{p_{k}}+X_{q_{k}},\left[X_{p_{k}}, X_{q_{k}}\right]\right]$ as in the proof of Lemma 4.6, Therefore we set

$$
\begin{aligned}
\overline{\bar{z}}_{k}=\overline{\bar{z}}_{k-1} & \circ e^{4 \tau\left(-\frac{\lambda_{k}}{2} X_{p_{k}}+Y\right)} \\
& \circ\left(e^{\tau\left(-\frac{\lambda_{k}}{2} X_{p_{k}}+Y\right)} \circ e^{\tau\left(-\frac{\lambda_{k}}{2} X_{q_{k}}+Y\right)} \circ e^{\tau\left(\frac{\lambda_{k}}{2} X_{p_{k}}+Y\right)} \circ e^{\tau\left(\frac{\lambda_{k}}{2} X_{q_{k}}+Y\right)}\right) \\
& \circ e^{4 \tau\left(\frac{\lambda_{k}}{2} X_{p_{k}}+Y\right)} \\
& \circ\left(e^{\tau\left(\frac{\lambda_{k}}{2} X_{p_{k}}+Y\right)} \circ e^{\tau\left(\frac{\lambda_{k}}{2} X_{q_{k}}+Y\right)} \circ e^{\tau\left(-\frac{\lambda_{k}}{2} X_{p_{k}}+Y\right)} \circ e^{\tau\left(-\frac{\lambda_{k}}{2} X_{q_{k}}+Y\right)}\right) \\
& \circ e^{4 \tau\left(-\frac{\lambda_{k}}{2} X_{q_{k}}+Y\right)} \\
& \circ\left(e^{\tau\left(-\frac{\lambda_{k}}{2} X_{p_{k}}+Y\right)} \circ e^{\tau\left(-\frac{\lambda_{k}}{2} X_{q_{k}}+Y\right)} \circ e^{\tau\left(\frac{\lambda_{k}}{2} X_{p_{k}}+Y\right)} \circ e^{\tau\left(\frac{\lambda_{k}}{2} X_{q_{k}}+Y\right)}\right) \\
& \circ e^{4 \tau\left(\frac{\lambda_{k}}{2} X_{q_{k}}+Y\right)} \\
& \circ\left(e^{\tau\left(\frac{\lambda_{k}}{2} X_{p_{k}}+Y\right)} \circ e^{\tau\left(\frac{\lambda_{k}}{2} X_{q_{k}}+Y\right)} \circ e^{\tau\left(-\frac{\lambda_{k}}{2} X_{p_{k}}+Y\right)} \circ e^{\tau\left(-\frac{\lambda_{k}}{2} X_{q_{k}}+Y\right)}\right)
\end{aligned}
$$

for $k=1, \ldots, m_{2}$ and, by (4.13), we have

$$
\overline{\bar{z}}_{k}=\overline{\bar{z}}_{k-1} \circ e^{32 \tau Y-\frac{1}{2} \tau^{3} \lambda_{k}^{3}\left[X_{p_{k}}+X_{q_{k}},\left[X_{p_{k}}, X_{q_{k}}\right]\right]-12 \tau^{2} \lambda_{k}\left[X_{p_{k}}+X_{q_{k}}, Y\right]} .
$$

Then, if we set $\bar{z}_{0}=\overline{\bar{z}}_{m_{2}}$, by the Campbell-Hausdorff formula, we get

$$
\bar{z}_{0}=\exp \left(\frac{9}{10} t Y+\sum_{1 \leq k \leq m_{2}} \tau^{2} \lambda_{k}^{2}\left[X_{p_{k}}, X_{q_{k}}\right]-10 \sum_{1 \leq k \leq m_{2}} \tau^{2} \lambda_{k}\left[X_{p_{k}}+X_{q_{k}}, Y\right]\right)(z) .
$$

Finally, we annihilate the last error term by setting

$\bar{z}_{k}=\bar{z}_{k-1} \circ\left(e^{\tau\left(10 \lambda_{k} X_{p_{k}}+Y\right)} \circ e^{\tau\left(-10 \lambda_{k} X_{p_{k}}+Y\right)}\right) \circ\left(e^{\tau\left(10 \lambda_{k} X_{q_{k}}+Y\right)} \circ e^{\tau\left(-10 \lambda_{k} X_{q_{k}}+Y\right)}\right)$,

for $k=1, \ldots, m_{2}$, and by (4.8) we have

$$
\bar{z}_{k}=\bar{z}_{k-1} \circ e^{4 \tau Y+10 \tau^{2} \lambda_{k}\left[X_{p_{k}}+X_{q_{k}}, Y\right]} .
$$

It is easy to check that

$$
\bar{z} \equiv \bar{z}_{m_{2}}=\exp \left(t Y+\sum_{1 \leq k \leq m_{2}} \tau^{2} \lambda_{k}^{2}\left[X_{p_{k}}, X_{q_{k}}\right]\right)(z),
$$

thus, by using (4.21), we infer

$$
\bar{x}^{(2)}=x^{(2)}+\sum_{1 \leq k \leq m_{2}} \tau^{2} \lambda_{k}^{2} \mathbf{e}_{m_{1}+k}+t b_{0} .
$$

We next choose $\lambda_{k}$ so that our claim $\bar{x}^{(2)}=-2 t b_{0}$ holds. Clearly this means $\tau^{2} \lambda_{k}^{2}=$ $-\left(3 t b_{0}+x\right)_{m_{1}+k}$, and the equation can be solved only when $\left(3 t b_{0}+x\right)_{m_{1}+k} \leq 0$. However, if this is not the case, we may exchange the role of $X_{p_{k}}$ and $X_{q_{k}}$ in (4.22) 
to obtain $\tau^{2} \lambda_{k}^{2}=\left(3 t b_{0}+x\right)_{m_{1}+k}$ instead. In both cases Theorem 1.1 gives

$$
u(\bar{z}) \leq \exp \left(c^{\prime}\left(1+\frac{\left|x^{(2)}+t b_{0}\right|}{t}\right)\right) u(z),
$$

and claim (4.18) follows.

Lemma 4.9. Let $z=(x, 4 t) \in \mathbb{R}^{N} \times \mathbb{R}^{+}$. There exist two constants $c>0, C>1$, only dependent on $L$, such that

$$
u(\bar{z}) \leq \exp \left(C\left(1+\frac{\left|x^{(1)}\right|^{2}}{t}\right)\right) u(z)
$$

for every non-negative solution $u$ to $L u=0$ in $\left.\mathbb{R}^{N} \times\right]$-ct, 4t], where

$$
\bar{z}=\left(\bar{x}^{(2)}+\bar{x}^{(3)}, 3 t\right)
$$

and (see the notations (4.5) -(4.6) )

$$
\begin{aligned}
\bar{x}^{(2)}= & x^{(2)}+t b_{0}-\frac{1}{2} \sum_{1 \leq p \leq m_{1}} x_{p} a_{1}^{p}\left(x^{(1)}\right), \\
\bar{x}^{(3)}= & x^{(3)}+\frac{t}{2} b_{1}\left(x^{(1)}\right) \\
& -\sum_{1 \leq p \leq m_{1}} x_{p}\left(\frac{1}{3} A_{2}^{p}\left(x^{(1)}\right)+A_{1}^{p}\left(x^{(2)}+\frac{t}{2} b_{0}-\frac{1}{3} \sum_{1 \leq q \leq m_{1}} x_{q} a_{1}^{q}\left(x^{(1)}\right)\right)\right) .
\end{aligned}
$$

Proof. We set

$$
z_{0}=z \circ e^{\tau\left(\lambda_{1} X_{1}+Y\right)} \circ e^{\tau\left(\lambda_{2} X_{2}+Y\right)} \circ \cdots \circ e^{\tau\left(\lambda_{m_{1}} X_{m_{1}}+Y\right)},
$$

for some $\lambda_{1}, \ldots, \lambda_{m_{1}}$ and $\tau>0$ to be suitably chosen. By the Campbell-Hausdorff formula, we have

$$
\begin{aligned}
z_{0} & =\exp \left(m_{1} \tau Y+\tau \sum_{1 \leq p \leq m_{1}} \lambda_{p} X_{p}+\tau^{2} \sum_{1 \leq k \leq m_{1}^{2}} c_{1, k} \lambda_{p_{k}} \lambda_{q_{k}}\left[X_{p_{k}}, X_{q_{k}}\right]\right. \\
& \left.+\tau^{3} \sum_{1 \leq k \leq m_{1}^{3}} c_{2, k} \lambda_{r_{k}} \lambda_{p_{k}} \lambda_{q_{k}}\left[X_{r_{k}},\left[X_{p_{k}}, X_{q_{k}}\right]\right]+\tau^{2} \sum_{1 \leq k \leq m_{1}} c_{3, k} \lambda_{k}\left[X_{k}, Y\right]\right)(z)
\end{aligned}
$$

for some constants $c_{1, k}, c_{2, k}, c_{3, k}$ (here we rearranged the sums to simplify the notations). Next we proceed as in the previous lemmas in order to remove the error terms. We set $\alpha_{k}=\sqrt{\left|c_{1, k}\right|}$ and

$z_{k}=z_{k-1} \circ e^{\tau\left(\alpha_{k} \lambda_{p_{k}} X_{p_{k}}+Y\right)} \circ e^{\tau\left(\alpha_{k} \lambda_{q_{k}} X_{q_{k}}+Y\right)} \circ e^{\tau\left(-\alpha_{k} \lambda_{p_{k}} X_{p_{k}}+Y\right)} \circ e^{\tau\left(-\alpha_{k} \lambda_{q_{k}} X_{q_{k}}+Y\right)}$

if $c_{1, k} \leq 0$, and

$z_{k}=z_{k-1} \circ e^{\tau\left(\alpha_{k} \lambda_{q_{k}} X_{q_{k}}+Y\right)} \circ e^{\tau\left(\alpha_{k} \lambda_{p_{k}} X_{p_{k}}+Y\right)} \circ e^{\tau\left(-\alpha_{k} \lambda_{q_{k}} X_{q_{k}}+Y\right)} \circ e^{\tau\left(-\alpha_{k} \lambda_{p_{k}} X_{p_{k}}+Y\right)}$, otherwise, for $k=1, \ldots, m_{1}^{2}$. By (4.20), we have

$$
\begin{aligned}
z_{k}= & \exp \left(4 \tau Y-\tau^{2} c_{1, k} \lambda_{p_{k}} \lambda_{q_{k}}\left[X_{p_{k}}, X_{q_{k}}\right]+2 \tau^{2} \alpha_{k}\left[\lambda_{p_{k}} X_{p_{k}}+\lambda_{q_{k}} X_{q_{k}}, Y\right]\right. \\
& \left.-\frac{1}{2} \tau^{3} c_{1, k} \alpha_{k} \lambda_{p_{k}} \lambda_{q_{k}}\left[\lambda_{p_{k}} X_{p_{k}}+\lambda_{q_{k}} X_{q_{k}},\left[X_{p_{k}}, X_{q_{k}}\right]\right]\right)\left(z_{k-1}\right) .
\end{aligned}
$$


Then if we let $\overline{\bar{z}}_{0}=z_{m_{1}^{2}}$, we get

$$
\begin{aligned}
\overline{\bar{z}}_{0}= & \exp \left(\left(m_{1}+4 m_{1}^{2}\right) \tau Y+\tau \sum_{1 \leq p \leq m_{1}} \lambda_{p} X_{p}\right. \\
& \left.+\tau^{3} \sum_{1 \leq k \leq m_{1}^{3}} c_{4, k} \lambda_{r_{k}} \lambda_{p_{k}} \lambda_{q_{k}}\left[X_{r_{k}},\left[X_{p_{k}}, X_{q_{k}}\right]\right]+\tau^{2} \sum_{1 \leq k \leq m_{1}} c_{5, k} \lambda_{k}\left[X_{k}, Y\right]\right)(z)
\end{aligned}
$$

for some constants $c_{4, k}, c_{5, k}$. Next we set $\beta_{k}=-\operatorname{sign}\left(c_{4, k}\right) \sqrt[3]{\left|c_{4, k}\right|}$ and

$$
\begin{aligned}
\overline{\bar{z}}_{k}= & \overline{\bar{z}}_{k-1} \circ e^{4 \tau\left(\frac{1}{4} \beta_{k} \lambda_{r_{k}} X_{r_{k}}+Y\right)} \\
& \circ\left(e^{\tau\left(\beta_{k} \lambda_{p_{k}} X_{p_{k}}+Y\right)} \circ e^{\tau\left(\beta_{k} \lambda_{q_{k}} X_{q_{k}}+Y\right)} \circ e^{\tau\left(-\beta_{k} \lambda_{p_{k}} X_{p_{k}}+Y\right)} \circ e^{\tau\left(-\beta_{k} \lambda_{q_{k}} X_{q_{k}}+Y\right)}\right) \\
& \circ e^{4 \tau\left(-\frac{1}{4} \beta_{k} \lambda_{r_{k}} X_{r_{k}}+Y\right)} \\
& \circ\left(e^{\tau\left(-\beta_{k} \lambda_{p_{k}} X_{p_{k}}+Y\right)} \circ e^{\tau\left(-\beta_{k} \lambda_{q_{k}} X_{q_{k}}+Y\right)} \circ e^{\tau\left(\beta_{k} \lambda_{p_{k}} X_{p_{k}}+Y\right)} \circ e^{\tau\left(\beta_{k} \lambda_{q_{k}} X_{q_{k}}+Y\right)}\right),
\end{aligned}
$$

for $k=1, \ldots, m_{1}^{3}$. By (4.13), we have

$$
\overline{\bar{z}}_{k}=\overline{\bar{z}}_{k-1} \circ e^{16 \tau Y-c_{4, k} \tau^{3} \lambda_{r_{k}} \lambda_{p_{k}} \lambda_{q_{k}}\left[X_{r_{k}},\left[X_{p_{k}}, X_{q_{k}}\right]\right]-4 \beta_{k} \tau^{2}\left[\lambda_{p_{k}} X_{p_{k}}+\lambda_{q_{k}} X_{q_{k}}+\lambda_{r_{k}} X_{r_{k}}, Y\right]} ;
$$

thus, if we let $\bar{z}_{0}=z_{m_{1}^{3}}$, by the Campbell-Hausdorff formula, we get

$$
\bar{z}_{0}=\exp \left(\left(m_{1}+4 m_{1}^{2}+16 m_{1}^{3}\right) \tau Y+\tau \sum_{1 \leq p \leq m_{1}} \lambda_{p} X_{p}+\tau^{2} \sum_{1 \leq k \leq m_{1}} c_{6, k} \lambda_{k}\left[X_{k}, Y\right]\right)(z)
$$

for some constants $c_{6, k}$. Finally, if we put

$$
\bar{z}_{k}=\bar{z}_{k-1} \circ e^{\tau\left(-c_{6, k} \lambda_{k} X_{k}+Y\right)} \circ e^{\tau\left(c_{6, k} \lambda_{k} X_{k}+Y\right)}, \quad k=1, \ldots, m_{1},
$$

by (4.8), we have

$$
\bar{z}_{k}=\bar{z}_{k-1} \circ e^{2 \tau Y-\tau^{2} c_{6, k} \lambda_{k}\left[X_{k}, Y\right]},
$$

and, by again using the Campbell-Hausdorff formula, we get

$$
\bar{z} \equiv \bar{z}_{m_{1}}=\exp \left(t Y+\tau \sum_{1 \leq p \leq m_{1}} \lambda_{p} X_{p}\right)(z)
$$

for $\tau=t\left(3 m_{1}+4 m_{1}^{2}+16 m_{1}^{3}\right)^{-1}$. Then we set $\lambda_{p}=-\frac{x_{p}}{\tau}$ so that $\bar{z} \in V_{1}^{\perp} \times \mathbb{R}$. With this choice of the $\lambda_{p}$ 's, it is straightforward to check that $\bar{x}^{(2)}$ and $\bar{x}^{(3)}$ are as in the statement.

Proof of Proposition 4.1. Since $L$ is invariant with respect to the left o-translations, it is not restrictive to assume $z_{0}=0$. We denote $t=\frac{s}{4}, x=x^{(1)}+x^{(2)}+w^{\prime}+w^{\prime \prime}$, and we apply Lemma 4.9 to the point $z=(x, 4 t)$, then Lemma 4.8 to the point $z=\left(\bar{x}^{(2)}+\bar{x}^{(3)}, 3 t\right)$, where $\bar{x}^{(2)}$ and $\bar{x}^{(3)}$ are as in the statement of Lemma 4.9. We next apply Lemma 4.6 to $z=\left(\bar{x}^{(3)}-2 t b_{0}, 2 t\right)$, and finally Lemma 4.5 to $z=$ $\left(\bar{w}^{\prime \prime}-t b_{0}, t\right)$ (recall the notation $\left.\bar{x}^{(3)}=\bar{w}^{\prime}+\bar{w}^{\prime \prime}\right)$. We thus get

$$
u(0) \leq \exp \left(C_{0}\left(1+\frac{\left|x^{(1)}\right|^{2}}{s}+\frac{\left|\bar{x}^{(2)}\right|}{s}+\frac{\left|\bar{w}^{\prime}\right|^{\frac{2}{3}}}{s}+\frac{\left|\bar{w}^{\prime \prime}\right|^{2}}{s^{3}}\right)\right) u(z),
$$


for a positive constant $C_{0}$. To complete the proof, we only need to estimate the norm of the vectors $x^{(2)}, \bar{w}^{\prime}$ and $\bar{w}^{\prime \prime}$. From the definition of $\bar{x}^{(2)}$ we infer

$$
\left|\bar{x}^{(2)}\right| \leq\left|x^{(2)}\right|+t\left|b_{0}\right|+c_{0}\left|x^{(1)}\right|^{2}
$$

for a suitable positive constant $c_{0}$; then (4.26) becomes

$$
u(0) \leq \exp \left(C_{1}\left(1+\frac{\left|x^{(1)}\right|^{2}}{s}+\frac{\left|x^{(2)}\right|}{s}+\frac{\left|\bar{w}^{\prime}\right|^{\frac{2}{3}}}{s}+\frac{\left|\bar{w}^{\prime \prime}\right|^{2}}{s^{3}}\right)\right) u(z) .
$$

Analogously, from the definition of $\bar{x}^{(3)}$ we get

$$
\left|\bar{x}^{(3)}-x^{(3)}\right| \leq c_{1} s\left|x^{(1)}\right|+c_{2}\left|x^{(1)}\right|^{3}+c_{3}\left|x^{(1)}\right|\left|x^{(2)}\right|,
$$

for three positive constants $c_{1}, c_{2}$ and $c_{3}$ depending on the operator $L$. Then we find

$$
u\left(z_{0}\right) \leq \exp \left(C\left(1+\frac{\left|x^{(1)}\right|^{6}}{s^{3}}+\frac{\left|x^{(1)}\right|^{2}\left|x^{(2)}\right|^{2}}{s^{3}}+\frac{\left|x^{(2)}\right|}{s}+\frac{\left|w^{\prime}\right|^{\frac{2}{3}}}{s}+\frac{\left|w^{\prime \prime}\right|^{2}}{s^{3}}\right)\right) u\left(z_{0} \circ z\right),
$$

and the claim follows.

Proof of Proposition 4.2. Under the assumptions $W^{\prime \prime}=\{0\}$ so that, arguing as in the proof of Proposition 4.1. we find

$$
u(0) \leq \exp \left(C_{1}\left(1+\frac{\left|x^{(1)}\right|^{2}}{s}+\frac{\left|x^{(2)}\right|}{s}+\frac{\left|\bar{x}^{(3)}\right|^{\frac{2}{3}}}{s}\right)\right) u(z),
$$

instead of (4.27). The claim follows from (4.28).

Proof of Proposition 4.3. In this case we have $V_{2}=W^{\prime}=\{0\}$ and $\bar{x}^{(3)}=x^{(3)}+$ $\frac{s}{2} b_{1}\left(x^{(1)}\right)$. Then (4.26) reads

$$
u(0) \leq \exp \left(C_{0}\left(1+\frac{\left|x^{(1)}\right|^{2}}{s}+\frac{\left|\bar{x}^{(3)}\right|^{2}}{s^{3}}\right)\right) u(z),
$$

and the claim plainly follows.

Proof of Proposition 4.4. According with the notations of Example 1.5, we have

$$
\begin{gathered}
\left\{(\xi, 0,0) \mid \xi \in \mathbb{R}^{m}\right\}=V_{1}, \\
\left\{(0, \eta, 0) \mid \eta \in \mathbb{R}^{n}\right\}=V_{2}+W^{\prime}, \\
\left\{(0,0, \omega) \mid \omega \in \mathbb{R}^{r}\right\}=W^{\prime \prime} .
\end{gathered}
$$

We also denote $x=(\xi, \eta, \omega)$. In our setting, by (1.23) we have that $a_{2}^{p}\left(x^{(1)}, x^{(2)}\right) \in$ $W^{\prime}$ in (4.5). Moreover, by (1.24), $b_{0}=0$ and $b_{1}\left(x^{(1)}\right) \in W^{\prime \prime}$ in (4.5). Furthermore from the linearity of $A_{1}^{p}$ and $A_{2}^{p}$ in (4.6), it follows that $A_{2}^{p}\left(x^{(1)}\right), A_{1}^{p}\left(x^{(2)}\right) \in W^{\prime}$ 
for any $x \in \mathbb{R}^{N}$. Then we use (4.27) as in the proof of Proposition 4.1, with

$$
\begin{aligned}
& \bar{w}^{\prime}=w^{\prime}-\sum_{1 \leq p \leq m_{1}} x_{p}\left(\frac{1}{3} A_{2}^{p}\left(x^{(1)}\right)+A_{1}^{p}\left(x^{(2)}-\frac{1}{3} \sum_{1 \leq q \leq m_{1}} x_{q} a_{1}^{q}\left(x^{(1)}\right)\right)\right), \\
& \bar{w}^{\prime \prime}=w^{\prime \prime}+\frac{s}{2} b_{1}\left(x^{(1)}\right) .
\end{aligned}
$$

This accomplishes the proof.

\section{Gaussian estimates}

In this section we prove Proposition 1.2 for Lie groups of step three by using the Harnack estimates of the previous section. We also give sharp Gaussian estimates for the operators in Examples 1.3, 1.4 and 1.5.

Proposition 5.1. Let $L$ be the parabolic operator (1.14) on a Carnot $\mathbb{G}$ group of step three and let $\Gamma$ be its fundamental solution. There exists a positive constant $C$ such that

$$
\Gamma(x, t) \geq \frac{C}{t^{\frac{Q-2}{2}}} \exp \left(-C \frac{|x|_{\mathbb{G}}^{2}}{t}\right), \quad \forall(x, t) \in \mathbb{R}^{N} \times \mathbb{R}^{+} .
$$

Proposition 5.2. Let $L$ be the Kolmogorov operator (1.16) on a group $\mathbb{K}$ of step three and let $\Gamma$ be its fundamental solution. There exists a positive constant $C$ such that

$$
\Gamma(x, t) \geq \frac{C}{t^{\frac{Q-2}{2}}} \exp \left(-C\left(\frac{\left|x^{(1)}\right|_{\mathbb{K}}^{2}}{t}+\frac{\left|x^{(3)}\right|_{\mathbb{K}}^{6}}{t^{3}}\right)\right), \quad \forall(x, t) \in \mathbb{R}^{N} \times \mathbb{R}^{+} .
$$

Proposition 5.3. Let $L$ be the operator in (1.22) on a linked group $\mathbb{L}=\mathbb{G} \triangle \mathbb{K}$ of step three and let $\Gamma$ be its fundamental solution. There exists a positive constant $C$ such that

$$
\begin{aligned}
\Gamma(x, y, w, t) \geq \frac{C}{t^{\frac{Q-2}{2}}} \exp \left(-C\left(\frac{|(x, y, 0)|_{\mathbb{L}}^{2}}{t}+\frac{|(0,0, w)|_{\mathbb{L}}^{6}}{t^{3}}\right)\right), & \\
& \forall(x, y, w, t) \in \mathbb{R}^{N} \times \mathbb{R}^{+} .
\end{aligned}
$$

Proof of Proposition 1.2. For $\xi \in \mathbb{R}^{N}$, we apply Proposition 4.1 to $u=\Gamma$ with $z_{0}=\left(0, \frac{c}{c+1}\right), T=\frac{1}{c+1}$ and $z=z_{0}^{-1} \circ(\xi, 1)$ (here $c$ is the constant in the statement of Proposition 4.1). Note that $z=(x, t)$ with $t=\frac{1}{c+1}$ and, by the triangular inequality,

$$
|x|_{\mathbb{G}} \leq\|z\|_{\mathbb{G}} \leq c_{0}\left(\|(\xi, 1)\|_{\mathbb{G}}+\left\|z_{0}^{-1}\right\|_{\mathbb{G}}\right) \leq C^{\prime}\left(|\xi|_{\mathbb{G}}+1\right)
$$

for some positive constant $C^{\prime}$ only depending on $c$ and $c_{0}$. Thus we find

$$
\Gamma(\xi, 1) \geq C \exp \left(-C|x|_{\mathbb{G}}^{6}\right) \Gamma\left(0, \frac{c}{c+1}\right) \geq C_{1} \exp \left(-C_{1}|\xi|_{\mathbb{G}}^{6}\right),
$$

where the constant $C_{1}$ does not depend on $\xi \in \mathbb{R}^{N}$. To prove the claim it is sufficient to use the homogeneity of the fundamental solution (see (1.4) ).

Proof of Proposition 5.1. We argue exactly as above by using Proposition 4.2 instead of Proposition 4.1 . 
Proof of Proposition 5.2. We proceed as in the proof of Proposition 1.2 and use Proposition 4.3. By (1.21) it is easy to check that $x=\xi$, since $z_{0}^{-1}=-z_{0}$ so that

$$
(x, t)=\left(0,-\frac{c}{c+1}\right) \circ(\xi, 1)=\left(\xi, \frac{1}{c+1}\right) \text {. }
$$

Then we obtain

$$
\Gamma(\xi, 1) \geq C_{1} \exp \left(-C_{1}\left(\left|\xi^{(1)}\right|^{2}+\left|\xi^{(3)}\right|^{2}\right)\right)
$$

instead of (5.4). We conclude as in the proof of Proposition 1.2, by using the homogeneity of $\Gamma$.

Proof of Proposition 5.3. For $(\xi, \eta, \phi) \in \mathbb{R}^{m} \times \mathbb{R}^{n} \times \mathbb{R}^{r}$, we argue again as above and use Proposition 4.4 with $z_{0}=\left(0,0,0, \frac{c}{c+1}\right)$ to get the following inequality:

$$
\Gamma(\xi, \eta, \omega, 1) \geq C \exp \left(-C\left(|(x, y, 0)|_{\mathbb{L}}^{2}+|w|^{2}\right)\right),
$$

where $(x, y, w, t)=z_{0}^{-1} \circ(\xi, \eta, \omega, 1)$. From (1.25) and (1.21) it follows that $z_{0}^{-1}=$ $-z_{0}$. Thus, again using (1.25), (1.21) and (1.26), we infer

$$
(x, y, w, t)=\left(0,0,0,-\frac{c}{c+1}\right) \circ(\xi, \eta, \omega, 1)=\left(\xi, \eta, \omega, \frac{1}{c+1}\right),
$$

and the claim follows.

\section{REFERENCES}

1. Georgios K. Alexopoulos, Sub-Laplacians with drift on Lie groups of polynomial volume growth, Mem. Am. Math. Soc. 739 (2002), 101 pp. (English). MR1878341 (2003c:22015)

2. D. G. Aronson and James Serrin, Local behavior of solutions of quasilinear parabolic equations, Arch. Rational Mech. Anal. 25 (1967), 81-122. MR0244638 (39:5952)

3. A. Bonfiglioli, E. Lanconelli, and F. Uguzzoni, Uniform Gaussian estimates of the fundamental solutions for heat operators on Carnot groups., Adv. Differ. Equ. 7 (2002), no. 10, 1153-1192 (English). MR.1919700 (2003f:35054)

4. G. B. Folland, Subelliptic estimates and function spaces on nilpotent Lie groups, Ark. Mat. 13 (1975), no. 2, 161-207. MR0494315 (58:13215)

5. David S. Jerison and Antonio Sánchez-Calle, Estimates for the heat kernel for a sum of squares of vector fields, Indiana Univ. Math. J. 35 (1986), no. 4, 835-854. MR88c:58064

6. Velimir Jurdjevic, Geometric control theory, Cambridge Studies in Advanced Mathematics, vol. 52, Cambridge University Press, Cambridge, 1997. MR 1425878 (98a:93002)

7. A. E. Kogoj and E. Lanconelli, An invariant Harnack inequality for a class of hypoelliptic ultraparabolic equations, Mediterr. J. Math. 1 (2004), 51-80. MR2088032 (2006b:35042)

8. _ One-side Liouville theorems for a class of hypoelliptic ultraparabolic equations, "Geometric Analysis of PDE and Several Complex Variables", Contemp. Math., vol. 368, Amer. Math. Soc., Providence, RI, 2005, pp. 305-312. MR2126477 (2005j:35025)

9. L. P. Kupcov, The fundamental solutions of a certain class of elliptic-parabolic second order equations, Differencial'nye Uravnenija 8 (1972), 1649-1660, 1716. MR0315290 (47:3839)

10. S. Kusuoka and D. Stroock, Applications of the Malliavin calculus. III, J. Fac. Sci. Univ. Tokyo Sect. IA Math. 34 (1987), no. 2, 391-442. MR0914028 (89c:60093)

11. E. Lanconelli and S. Polidoro, On a class of hypoelliptic evolution operators, Rend. Sem. Mat. Univ. Politec. Torino 52 (1994), no. 1, 29-63, Partial differential equations, II (Turin, 1993). MR:1289901 (95h:35044)

12. Ermanno Lanconelli, Andrea Pascucci, and Sergio Polidoro, Linear and nonlinear ultraparabolic equations of Kolmogorov type arising in diffusion theory and in finance, Nonlinear problems in mathematical physics and related topics, II, Int. Math. Ser. (N.Y.), vol. 2, Kluwer/Plenum, New York, 2002, pp. 243-265. MR.1972000(2004c:35238)

13. Alexander Nagel, Elias M. Stein, and Stephen Wainger, Balls and metrics defined by vector fields. I. Basic properties, Acta Math. 155 (1985), no. 1-2, 103-147. MR0793239 (86k:46049) 
14. A. Pascucci and S. Polidoro, On the Harnack inequality for a class of hypoelliptic evolution equations, Trans. Amer. Math. Soc. 356 (2004), 4383-4394. MR 2067125

15. Andrea Pascucci and Sergio Polidoro, A Gaussian upper bound for the fundamental solutions of a class of ultraparabolic equations, J. Math. Anal. Appl. 282 (2003), no. 1, 396-409. MR 2000352 (2004j:35167)

16. S. Polidoro, On a class of ultraparabolic operators of Kolmogorov-Fokker-Planck type, Matematiche (Catania) 49 (1994), no. 1, 53-105. MR1386366 (97a:35133)

17. _ A global lower bound for the fundamental solution of Kolmogorov-Fokker-Planck equations, Arch. Rational Mech. Anal. 137 (1997), no. 4, 321-340. MR.1463798 (98g:35123)

18. Linda Preiss Rothschild and E. M. Stein, Hypoelliptic differential operators and nilpotent groups, Acta Math. 137 (1976), no. 3-4, 247-320. MR0436223 (55:9171)

19. L. Saloff-Coste and D. W. Stroock, Opérateurs uniformément sous-elliptiques sur les groupes de Lie, J. Funct. Anal. 98 (1991), no. 1, 97-121. MR1111195 (92k:58264)

20. N. Th. Varopoulos, L. Saloff-Coste, and T. Coulhon, Analysis and geometry on groups, Cambridge Tracts in Mathematics, vol. 100, Cambridge University Press, Cambridge, 1992. MR.1218884 (95f:43008)

Dipartimento di Matematica, Università di Bologna, Piazza di Porta S. Donato 5, 40126 Bologna, ItAly

E-mail address: pascucci@dm.unibo.it

Dipartimento di Matematica, Università di Bologna, Piazza di Porta S. Donato 5, 40126 Bologna, Italy

E-mail address: polidoro@dm.unibo.it 